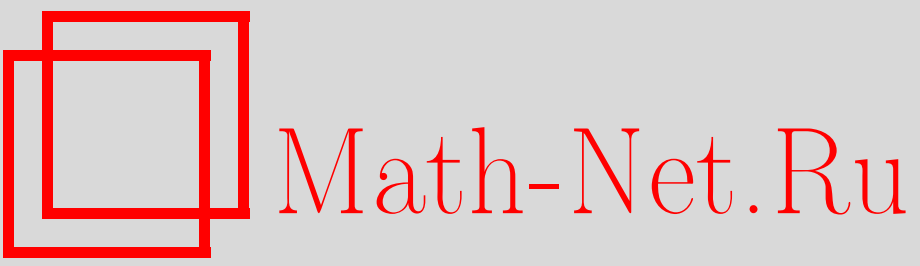

С. В. Демидов, С. Л. Дубовский, В. А. Рубаков, С. М. Сибиряков, Солитоны в калибровочной теории на некоммутативном цилиндре, ТМФ, 2004, том 138, номер 2, 319-337

DOI: https://doi.org/10.4213/tmf18

Использование Общероссийского математического портала Math-Net.Ru подразумевает, что вы прочитали и согласны с пользовательским соглашением

http://www.mathnet.ru/rus/agreement

Параметры загрузки:

IP: 54.224 .187 .69

26 апреля 2023 г., 16:56:36 


\author{
ТЕОРЕТИЧЕСКАЯ \\ И МАТЕМАТИЧЕСКАЯ \\ ФИЗИКА \\ Том 138, № 2 \\ февраль, 2004
}

(C) 2004 г. $\quad$ С.В. Демидов* , С. Л. Дубовский ${ }^{\dagger}$, В. А. Рубаков ${ }^{\dagger}$, С. М. Сибиряков ${ }^{\dagger}$

\title{
СОЛИТОНЫ В КАЛИБРОВОЧНОЙ ТЕОРИИ НА НЕКОММУТАТИВНОМ ЦИЛИНДРЕ
}

Метод генерации решений, предложенный изначально для калибровочных теорий на некоммутативной плоскости, обобщен на случай некоммутативного цилиндра. С этой целью построены операторы частичной изометрии и полный набор ортогональных проекторов в алгебре цилиндра $\mathcal{A}_{\mathrm{C}}$, а также изоморфизм между свободным модулем $\mathcal{A}_{\mathrm{C}}$ и его прямой суммой с фоковским модулем на цилиндре $\mathcal{A}_{\mathrm{C}} \oplus \mathcal{F}_{\mathrm{C}}$. В явном виде построен солитон в калибровочной теории и найден спектр малых возмущений над ним.

Ключевые слова: некоммутативная теория поля, калибровочная теория, солитоны.

\section{1. ВВЕДЕНИЕ}

В последнее время теории поля на некоммутативных пространствах вызвали значительный интерес (недавние обзоры и ссылки на предыдущие работы можно найти, например, в [1]-[4]). Одна из причин этого интереса состоит в том, что некоммутативная теория Янга-Миллса возникает как эффективное низкоэнергетическое описание теории струн во внешнем $B$-поле в определенном пределе [5]. Особое значение имеет изучение солитонов в некоммутативных теориях. Многие свойства этих солитонов аналогичны свойствам $D$-бран; замечательно, что правильно воспроизводятся величины натяжения $D$-бран, а спектр флуктуаций вокруг некоммутативных солитонов согласуется качественно и количественно со спектром открытых струн на фоне $D$-бран [6], [7].

Наиболее изученным примером некоммутативной теории поля является теория на некоммутативной плоскости (в более общем случае на некоммутативном пространстве $\left.\mathbb{R}^{2 n}\right)$. Алгебра функций на некоммутативной плоскости $\mathcal{A}_{\mathrm{P}}$ порождается двумя координатами $\hat{x}_{1} \equiv \hat{x}$ и $\hat{x}_{2} \equiv \hat{p}$, подчиняюшимися следуюшему коммутационному соотношению:

$$
[\hat{x}, \hat{p}]=i \theta
$$

\footnotetext{
* Московский физико-технический институт, Долгопрудный, Московская обл., Россия. E-mail: demidov@ms2.inr.ac.ru

${ }^{*}$ Институт ядерных исследований РАН, Москва, Россия. E-mail: sergd@ms2.inr.ac.ru, rubakov@ms2.inr.ac.ru, sibir@ms2.inr.ac.ru
} 
Эта алгебра изоморфна алгебре операторов квантовой механики системы с одной степенью свободы.

В пределе сильной некоммутативности, $\theta \rightarrow \infty$, солитоны в чисто скалярной теории выражаются в терминах проекторов $P$ в алгебре $\mathcal{A}_{\mathrm{P}}[8]$, удовлетворяюших соотношению

$$
P * P=P,
$$

где $*$ обозначает умножение в $\mathcal{A}_{\mathrm{P}}$. Для калибровочной теории на некоммутативной плоскости был предложен метод генерации решений [7], [9], [2], позволяюший получать новые точные решения, начиная с вакуумного. В этом методе используются операторы частичной изометрии - элементы $S$ алгебры $\mathcal{A}_{\mathrm{P}}$ со следующими свойствами:

$$
S S^{+}=1, \quad S^{+} S=1-P
$$

где $P$ - проектор. Таким образом, солитоны в некоммутативной калибровочной теории, получаюшиеся при помоши метода генерации решений, также определяются проекторами в алгебре $\mathcal{A}_{\mathrm{P}}$.

Как показано в работах [1], метод генерации решений тесно связан с наличием изоморфизма между свободным модулем над алгеброй $\mathcal{A}_{\mathrm{P}}$ и прямой суммой $\mathcal{F}_{\mathrm{P}} \oplus \mathcal{A}_{\mathrm{P}}$ фоковского модуля $\mathcal{F}_{\mathrm{P}}$ (которьй есть не что иное, как гильбертово пространство состояний гармонического осциллятора) и свободного модуля. Однако в случае некоммутативной плоскости использование формального алгебраического языка проективных модулей может показаться излишним, так как метод генерации решений в том виде, в котором он был предложен изначально [7], [9], [2], достаточно прозрачен сам по себе.

Следуюшими по сложности примерами некоммутативных пространств являются некоммутативные цилиндр и тор. Соответствуюшие алгебры будут обозначаться в дальнейшем $\mathcal{A}_{\mathrm{C}}$ и $\mathcal{A}_{\mathrm{T}}$. В случае калибровочной теории на торе построить солитон в терминах связности на $\mathcal{A}_{\mathrm{T}}$ оказывается затруднительно (насколько нам известно, к настоящему времени не построено ни одного явного солитонного решения). Построить солитон в некоммутативной калибровочной теории по-прежнему удается, если воспринимать его как связность на прямой сумме фоковского и свободного модулей $\mathcal{F}_{\mathrm{T}} \oplus \mathcal{A}_{\mathrm{T}}[10]$. Этот подход позволяет вычислить спектр малых возмушений вокруг солитона [10], который согласуется со спектром струн на фоне системы $D 0-D 2$-бран, компактифицированной в данном случае на тор. Однако, вследствие того что $\mathcal{F}_{\mathrm{T}} \oplus \mathcal{A}_{\mathrm{T}}$ и $\mathcal{A}_{\mathrm{T}}$ не изоморфны [1], связность на $\mathcal{F}_{\mathrm{T}} \oplus \mathcal{A}_{\mathrm{T}}$ не индуцирует связность на свободном модуле $\mathcal{A}_{\mathrm{T}}$, и метод генерации решений не может быть сформулирован.

В данной работе мы найдем и изучим точные классические решения в $U(1)$-калибровочной теории на некоммутативном цилиндре (описание скалярных солитонов на некоммутативном цилиндре в пределе больших $\theta$ приведено в [11], альтернативные подходы к некоммутативному шилиндру рассмотрены в [12]-[14]). В частности, мы обсудим вопрос о сушествовании метода генерации решений в этом случае. Мы дадим утвердительный ответ на этот вопрос и приведем явное солитонное решение непосредственно 
в терминах связности на алгебре $\mathcal{A}_{\mathrm{C}}$. В отличие от случая некоммутативной плоскости, алгебраический формализм, описанный в [1], оказывается наиболее адекватным для калибровочной теории на некоммутативном цилиндре.

Статья построена следуюшим образом. В разделе 2 мы даем описание алгебры $\mathcal{A}_{\mathrm{C}}$ функций на некоммутативном цилиндре как подалгебры алгебры некоммутативной плоскости $\mathcal{A}_{\mathrm{P}}$, вводим базис в фоковском пространстве $\mathcal{F}_{\mathrm{P}}$, удобный для построения проекторов и операторов частичной изометрии в алгебре цилиндра $\mathcal{A}_{\mathrm{C}}$, а также даем описание связностей на фоковском и свободном модулях над этой алгеброй. В разделе 3 мы описываем метод генерации решений для случая некоммутативного цилиндра и приводим явное построение солитона в калибровочной теории в терминах связности на свободном модуле $\mathcal{A}_{\mathrm{C}}$. В разделе 4 мы изучаем спектр флуктуаций вокруг этого солитона, используя его представление в виде связности на прямой сумме $\mathcal{A}_{\mathrm{C}} \oplus \mathcal{F}_{\mathrm{C}}$. В разделе 5 обсуждаются полученные результаты.

\section{2. НЕКОММУТАТИВНЫЙ ЦИЛИНДР}

2.1. Алгебра, проекторы, операторы частичной изометрии. В коммутативной геометрии алгебра функций на цилиндре радиуса $R$ с координатами $(x, p)$ может быть определена как подалгебра функций на плоскости, инвариантных относительно сдвигов на фиксированное расстояние:

$$
A(x, p)=A(x+2 \pi R, p) .
$$

На некоммутативной плоскости с координатами $(\hat{x}, \hat{p})$ бесконечно малые сдвиги порождаются внутренними дифференцированиями:

$$
\frac{\partial \hat{A}}{\partial \hat{x}}=\frac{i}{\theta}[\hat{p}, \hat{A}], \quad \frac{\partial \hat{A}}{\partial \hat{p}}=-\frac{i}{\theta}[\hat{x}, \hat{A}] .
$$

Следовательно, обобщение соотношения (2) на некоммутативный случай состоит в определении алгебры $\mathcal{A}_{\mathrm{C}}$ функций на некоммутативном цилиндре как подалгебры алгебры $\mathcal{A}_{\mathrm{P}}$, состоящей из функций, коммутируюших с оператором конечных сдвигов вдоль направления $x$ :

$$
\hat{A} \in \mathcal{A}_{\mathrm{C}}: e^{-2 \pi i R \hat{p} / \theta} \hat{A} e^{2 \pi i R \hat{p} / \theta}=\hat{A} .
$$

Генераторами алгебры $\mathcal{A}_{\mathrm{C}}$ являются $\hat{p}$ и $e^{i \hat{x} / R}$; координата $\hat{x}$, очевидно, не принадлежит $\mathcal{A}_{\mathrm{C}}$. Операции дифференцирования (3) сохраняют условие (4); таким образом, соотношения (3) дают определение производных на некоммутативном цилиндре. Заметим, что дифференцирование по координате $\hat{p}$ не является внутренней производной в алгебpe $\mathcal{A}_{\mathrm{C}}$.

Алгебра $\mathcal{A}_{\mathrm{P}}$ может рассматриваться как алгебра операторов, действуюших в гильбертовом пространстве функций, зависяших от одной переменной. В дальнейшем нам

6 Теоретическая и математическая физика, т. 138, № 2, 2004 г. 
будет удобно работать в $p$-представлении. Любой оператор $\hat{A}$ однозначно определяется своим ядром $A\left(p, p^{\prime}\right)$ :

$$
(\hat{A} \psi)(p)=\int d p^{\prime} A\left(p, p^{\prime}\right) \psi\left(p^{\prime}\right),
$$

где $\psi(p)$ - произвольная функция. Из условия (4) следует, что оператор принадлежит подалгебре $\mathcal{A}_{\mathrm{C}}$ тогда и только тогда, когда его ядро удовлетворяет соотношению

$$
A\left(p, p^{\prime}\right)=A\left(p, p^{\prime}\right) e^{-2 \pi i R\left(p-p^{\prime}\right) / \theta}
$$

или, что эквивалентно,

$$
A\left(p, p^{\prime}\right)=\sum_{q \in \mathbb{Z}} a(p, q) \delta\left(\frac{R p}{\theta}-\frac{R p^{\prime}}{\theta}-q\right)
$$

с произвольными коэффициентными функциями $a(p, q)$, зависяшими от одной непрерывной и одной дискретной переменной.

Для того чтобы построить теорию поля на некоммутативном цилиндре, необходимо определить след $\operatorname{Tr}_{\mathrm{C}}$ в алгебре $\mathcal{A}_{\mathrm{C}}$. Этот след является некоммутативным обобшением интеграла на цилиндре. Он не может быть определен просто как след $\operatorname{Tr}$ в алгебре $\mathcal{A}_{\mathrm{P}}$. Действительно, из представления (5) ясно, что след $\operatorname{Tr}$ расходится для элементов, лежаших в $\mathcal{A}_{\mathrm{C}}$. Это неудивительно: в коммутативном случае результат тоже был бы бесконечен, если бы функции на цилиндре воспринимались как периодические функции на плоскости и интеграл по цилиндру определялся бы как интеграл по всей плоскости. Вместо этого интеграл по цилиндру определяется как интеграл от периодической функции по периоду. Способ обобщить это определение на некоммутативный случай станет ясен, если вспомнить, что алгебра $\mathcal{A}_{\mathrm{C}}$ порождена двумя элементами $\hat{p}$ и $e^{i \hat{x} / R}$, т.е. каждый элемент алгебры имеет вид

$$
\hat{A}=\sum_{q=-\infty}^{\infty} a(\hat{p}, q) e^{i q \hat{x} / R}
$$

(На самом деле коэффициенты Фурье $a(\hat{p}, q)$ совпадают с коэффициентными функциями, возникаюшими в выражении (5) для ядра оператора $\hat{A}$.) Определим $\operatorname{Tr}_{\mathrm{C}} \hat{A}$ как интеграл от нулевой фурье-компоненты оператора $\hat{A}$ :

$$
\operatorname{Tr}_{\mathrm{C}} \hat{A} \equiv \int_{-\infty}^{\infty} a(p, 0) d p
$$

Легко проверить, что $\operatorname{Tr}_{\mathrm{C}}$ обладает всеми необходимыми свойствами операции следа.

Используя этот след, запишем вейлевский символ оператора (6):

$$
f_{\hat{A}}(x, p)=\frac{1}{2 \pi} \sum_{n} \int d \tau e^{-i n x / R-i \tau R p / \theta} \operatorname{Tr}_{\mathrm{C}}\left[\hat{A}(\hat{x}, \hat{p}) e^{i(n \hat{x} / R+\tau \hat{p} R / \theta)}\right] .
$$


Очевидно, этот символ периодичен по переменной $x$ с периодом $2 \pi R$. Обратное преобразование имеет вид

$$
\hat{A}(\hat{x}, \hat{p})=\frac{1}{(2 \pi)^{2}} \sum_{m} \int d \sigma e^{-i(m \hat{x} / R+\sigma R \hat{p} / \theta)} \tilde{f}_{\hat{A}}(m, \sigma),
$$

где

$$
\tilde{f}_{\hat{A}}(m, \sigma)=\int_{0}^{2 \pi R} d x \int d p e^{i(m x / R+\sigma R p / \theta)} f_{\hat{A}}(x, p) .
$$

Непосредственно можно проверить следуюшую связь между следом оператора и интегралом от его вейлевского символа:

$$
2 \pi \theta \operatorname{Tr}_{\mathrm{C}} \hat{A}=\int d x d p f_{\hat{A}}(x, p) .
$$

Это соотношение имеет ту же форму, что и в случае некоммутативной плоскости, - именно такую, которая нужна для построения теории поля на некоммутативном цилиндре.

Построим теперь проекторы и операторы частичной изометрии в алгебре $\mathcal{A}_{\mathrm{C}}$ некоммутативного цилиндра. В гильбертовом пространстве функций $\psi(p)$ удобно выбрать ортонормированный базис с определенными свойствами. А именно, элементы базиса $|n, m\rangle$ пронумерованы двумя числами $n=0,1,2, \ldots$ и $m=0, \pm 1, \pm 2, \ldots$ Определяющим свойством этого базиса является следующий закон преобразования при сдвигах на конечное расстояние:

$$
e^{2 \pi i R \hat{p} / \theta}|n, m\rangle=|n, m+1\rangle .
$$

Используя соотношение (4), находим, что последнее свойство и условие ортонормированности приводят к тому, что любой элемент алгебры $\mathcal{A}_{\mathrm{C}}$ может быть представлен в следуюшем виде:

$$
\hat{A}=\sum_{n, n^{\prime}, m, l} C_{n, n^{\prime}}^{l}|n, m\rangle\left\langle m+l, n^{\prime}\right|,
$$

где $C_{n, n^{\prime}}^{l}$ - произвольные коэффициенты. Теперь можно построить следуюший набор проекторов:

$$
P_{i}=\sum_{m}|i, m\rangle\langle m, i| .
$$

Их вид согласуется с обшим выражением (11), т.е. они принадлежат алгебре некоммутативного цилиндра. Эти проекторы взаимно ортогональны:

$$
P_{i} P_{j}=\delta_{i j} P_{i},
$$

и образуют полный набор в пространстве проекторов в алгебре $\mathcal{A}_{\mathrm{C}}$. Для того чтобы убедиться в последнем свойстве, проверим, что не сушествует ненулевого проектора $P \in$ $\mathcal{A}_{\mathrm{C}}$ такого, что для всех $i$

$$
P P_{i}=0 .
$$


Для любого проектора $P$ и базисной функции $|i, m\rangle$ имеем

$$
\| P|i, m\rangle \|^{2}=\operatorname{Tr}(P|i, m\rangle\langle m, i|) \geqslant 0 .
$$

Если $P \in \mathcal{A}_{\mathrm{C}}$, то в соответствии с равенствами (4) и (10) след в соотношении (14) не зависит от $m$. Следовательно, можно записать

$$
\| P|i, m\rangle \|^{2}=\frac{1}{2 N+1} \sum_{m=-N}^{m=N} \operatorname{Tr}(P|i, m\rangle\langle i, m|) .
$$

Из требования (13) следует, что входящая в это выражение сумма стремится к нулю, когда $N \rightarrow \infty$. Таким образом, любой проектор $P \in \mathcal{A}_{\mathrm{C}}$, удовлетворяющий требованию (13), аннигилирует все базисные функции $|i, m\rangle$ и, следовательно, равен нулю.

Не составляет труда построить набор операторов частичной изометрии, аналогичных оператору сдвига базиса в случае плоскости,

$$
S_{i}^{\mathrm{C}}=\sum_{m, n}|n, m\rangle\langle m, n+i|
$$

со свойством

$$
S_{i}^{\mathrm{C}} S_{i}^{\mathrm{C}+}=1, \quad S_{i}^{\mathrm{C}+} S_{i}^{\mathrm{C}}=1-P_{i} .
$$

Эти операторы тоже имеют вид (11) и, следовательно, принадлежат алгебре $\mathcal{A}_{\mathrm{C}}$.

Существование ортонормированного базиса $|n, m\rangle$ со свойством (10) далеко не очевидно. Докажем явным построением, что такой базис действительно сушествует. Из свойства (10) следует, что базисные элементы $|n, m\rangle$ имеют в $p$-представлении следующий вид:

$$
|n, m\rangle=\xi_{n+1}(p) e^{2 \pi i R m p / \theta}
$$

в то время как из ортонормированности и полноты набора $\{|n, m\rangle\}$ вытекает

$$
\int d p \xi_{n}(p) \xi_{n^{\prime}}(p) e^{2 \pi i R p\left(m-m^{\prime}\right) / \theta}=\delta_{n n^{\prime}} \delta_{m m^{\prime}}
$$

и

$$
\sum_{n, m} \xi_{n}(p) \xi_{n}\left(p^{\prime}\right) e^{2 \pi i R\left(p-p^{\prime}\right) m / \theta}=\delta\left(p-p^{\prime}\right) .
$$

Далее, пусть $\chi_{n}(\lambda)$ - произвольный ортонормированный базис в пространстве функций на интервале $\lambda \in[-\pi, \pi]$ такой, что $\chi_{n}(-\pi)=\chi_{n}(\pi)=0$. Тогда можно непосредственно проверить, что функции

$$
\xi_{n}(p)=\sqrt{\frac{R}{\theta}} \int_{-\pi}^{\pi} d \lambda \chi_{n}(\lambda) e^{2 \pi i R p \lambda / \theta}
$$


обладают требуемыми свойствами (17) и (18). Таким образом, любой вспомогательньй базис $\chi_{n}(\lambda)$ определяет базис $|n, m\rangle$, удовлетворяюший соотношению (10).

Относительно простой пример функций $\xi_{n}(p)$ дается следуюшим выбором вспомогательного базиса:

$$
\chi_{n}(\lambda)=\frac{1}{\sqrt{\pi}} \sin \frac{(\pi+\lambda)(n+1)}{2} .
$$

Соответствуюшие функции $\xi_{n}(p)$ имеют вид

$$
\begin{aligned}
\xi_{2 k} & =\sqrt{\frac{2 R}{\theta}} \frac{k}{\pi} \frac{\sin \pi R p / \theta}{(R p / \theta)^{2}-k^{2}}, \\
\xi_{2 k+1} & =\sqrt{\frac{2 R}{\theta}} \frac{(k+1 / 2)}{\pi} \frac{\cos \pi R p / \theta}{(R p / \theta)^{2}-(k+1 / 2)^{2}},
\end{aligned}
$$

где $k=0,1,2, \ldots$ Используя равенства $(16),(20)$ и (21), можно явно построить проекторы (12) и операторы частичной изометрии (15).

2.2. Модули и эндоморфизмы на некоммутативном цилиндре. Некоммутативными аналогами векторных расслоений и связностей (калибровочных полей) на этих расслоениях являются проективные модули (см., например, обзоры [1], [3]). В предыдушем разделе мы построили полный набор проекторов в алгебре $\mathcal{A}_{\mathrm{C}}$. Из этого построения вытекает, что, так же как и в случае некоммутативной плоскости, произвольный проективный модуль над $\mathcal{A}_{\mathrm{C}}$ разлагается в прямую сумму некоторого числа свободных и фоковских модулей. Опишем эти модули подробно.

Элементами (правого) свободного модуля являются элементы самой алгебры $\mathcal{A}_{\mathrm{C}}$, причем алгебра действует на этом модуле посредством умножения справа. Произвольная связность на этом модуле может быть представлена в виде

$$
\nabla_{i}=\partial_{i}+i u_{i}
$$

где

$$
\partial_{1}=\frac{\partial}{\partial \hat{x}}, \quad \partial_{2}=\frac{\partial}{\partial \hat{p}},
$$

а $u_{i}$ принадлежат алгебре $\operatorname{End}_{\mathcal{A}_{\mathrm{C}}}\left(\mathcal{A}_{\mathrm{C}}\right)$ эндоморфизмов свободного модуля, т.е., как и в случае некоммутативной плоскости, $u_{i}$ - это произвольные элементы алгебры $\mathcal{A}_{\mathrm{C}}$, действуюшие на свободный модуль умножением слева. Кривизна $F_{12}$ связности $\nabla_{i}$ определяется стандартным образом:

$$
F_{12}=\left[\nabla_{1}, \nabla_{2}\right] .
$$

Очевидно, что "каноническая" связность $\partial_{i}$ на свободном модуле имеет нулевую кривизну.

Элементами (правого) фоковского модуля $\mathcal{F}_{\mathrm{C}}$ на некоммутативном цилиндре являются бра-векторы $\langle f|$ фоковского модуля некоммутативной плоскости со стандартным действием на них операторов $\hat{A} \in \mathcal{A}_{\mathrm{C}} \subset \mathcal{A}_{\mathrm{P}}$ :

$$
\langle f| \hat{A}=\left(\hat{A}^{+}|f\rangle\right)^{+} .
$$


Этот модуль обладает связностью постоянной кривизны $\nabla_{i}^{0}$ со следуюшими компонентами:

$$
\nabla_{1}^{0}(\langle f|)=-\frac{i}{\theta}\langle f| \hat{p}, \quad \nabla_{2}^{0}(\langle f|)=\frac{i}{\theta}\langle f| \hat{x}
$$

Кривизна этой связности равна

$$
F_{12}=\left[\nabla_{1}^{0}, \nabla_{2}^{0}\right]=-\frac{i}{\theta}
$$

Произвольная связность на фоковском модуле может быть представлена в виде

$$
\nabla_{i}=\nabla_{i}^{0}+i z_{i}
$$

где $z_{i}$ принадлежит алгебре $\operatorname{End}_{\mathcal{A}_{\mathrm{C}}}\left(\mathcal{F}_{\mathrm{C}}\right)$ эндоморфизмов модуля $\mathcal{F}_{\mathrm{C}}$, т.е. калибровочные поля $z_{i}$ на фоковском модуле имеют следуюший вид:

$$
z_{i}=\sum_{n} z_{i}(n) e^{2 \pi i n R \hat{p} / \theta}
$$

где $z_{i}(n)$ - некоторые числа. Заметим, что, в отличие от случая некоммутативной плоскости, где на фоковском модуле возможны лиш постоянные калибровочные поля, $z_{i}$ в обшем случае не постоянны.

Ниже нам встретится также прямая сумма $\mathcal{F}_{\mathrm{C}} \oplus \mathcal{A}_{\mathrm{C}}$ фоковского и свободного модулей. Фиксируем обозначения, записав ее элементы в виде столбца

$$
\left(\begin{array}{c}
\langle f| \\
\hat{A}
\end{array}\right)
$$

Произвольная связность на таком модуле может быть записана в виде

$$
\nabla_{i}=\widetilde{\nabla}_{i}^{0}+i\left(\begin{array}{cc}
z_{i} & \psi_{i} \\
\psi_{i}^{*} & v_{i}
\end{array}\right)
$$

где

$$
\widetilde{\nabla}_{1}^{0}=\left(\begin{array}{cc}
-\frac{i}{\theta} \hat{p} & 0 \\
0 & \partial_{1}
\end{array}\right), \quad \widetilde{\nabla}_{2}^{0}=\left(\begin{array}{cc}
\frac{i}{\theta} \hat{x} & 0 \\
0 & \partial_{2}
\end{array}\right)
$$

Здесь $v_{i} \in \operatorname{End}_{\mathrm{C}}\left(\mathcal{A}_{\mathrm{C}}\right)$, в то время как $\psi$ и $\psi^{*}$ являются гомоморфизмами из свободного модуля $\mathcal{A}_{\mathrm{C}}$ в фоковский модуль $\mathcal{F}_{\mathrm{C}}$ и обратно. Эти гомоморфизмы могут быть заданы при помощи векторов $\left\langle\psi_{i}\right|$ и $\left|\psi_{i}\right\rangle$ следующим образом:

$$
\psi_{i}(\hat{A})=\left\langle\psi_{i}\right| \hat{A}, \quad \psi_{i}^{*}(\langle f|)=\sum_{m} e^{2 \pi i m R \hat{p} / \theta}\left|\psi_{i}\right\rangle\langle f| e^{-2 \pi i m R \hat{p} / \theta},
$$

где $\hat{A} \in \mathcal{A}_{\mathrm{C} \text { и }}\langle f| \in \mathcal{F}_{\mathrm{C}}$. 


\section{3. МЕТОД ГЕНЕРАЦИИ РЕШЕНИЙ НА НЕКОММУТАТИВНОМ ЦИЛИНДРЕ}

Рассмотрим $U(1)$-калибровочную теорию на некоммутативном цилиндре. Действие имеет вид

$$
S=\frac{\pi \theta}{2 g^{2}} \int d t \operatorname{Tr}_{\mathrm{C}}\left(F^{\mu \nu} F_{\mu \nu}\right)
$$

где $\mu, \nu=0,1,2$. Напряженность поля $F_{\mu \nu}$ определена стандартным образом:

$$
F_{\mu \nu}=\left[\nabla_{\mu}, \nabla_{\nu}\right]
$$

где $\nabla_{i}, \quad i=1,2$, определены соотношением $(22)$ и $\nabla_{0}=\partial_{t}+i u_{0}$. Компоненты калибровочного поля $u_{\mu}$ являются элементами алгебры $\mathcal{A}_{\mathrm{C}}$, действующими на свободньй модуль как умножение слева. В обшем случае эти поля зависят от времени. В этом разделе мы будем искать статические решения уравнений поля в калибровке $u_{0}=0$. Тогда статические уравнения поля имеют обычный вид:

$$
\left[\nabla_{i},\left[\nabla_{i}, \nabla_{j}\right]\right]=0
$$

Цель данного раздела состоит в том, чтобы сформулировать метод генерации решений в $U(1)$-калибровочной теории на некоммутативном цилиндре, аналогичный методу, предложенному для случая некоммутативной плоскости в работах [7], [9], [2].

Напомним, как выглядит метод генерации решений в случае некоммутативной плоскости [7], [9], [2]. Поскольку производные (23) являются в этом случае внутренними дифференцированиями алгебры $\mathcal{A}_{\mathrm{P}}$ (ср. с (3)), можно ввести следующие операторы:

$$
C_{1}=\frac{i}{\theta} \hat{p}+i u_{1}, \quad C_{2}=-\frac{i}{\theta} \hat{x}+i u_{2}
$$

Тогда статические уравнения поля (28) принимают вид

$$
\left[C_{i},\left[C_{i}, C_{j}\right]\right]=0
$$

Разница между уравнениями (28) и (29) состоит в том, что в первое входят коэффициенты связности на свободном модуле над алгеброй $\mathcal{A}_{\mathrm{P}}$, в то время как последнее содержит элементы самой алгебры. Ключевое наблюдение заключается в следуюшем: если элементы $C_{i}^{0}$ являются решениями уравнения (29), а $S$ - произвольный элемент алгебры $\mathcal{A}_{\mathrm{P}}$, удовлетворяющий соотношению $S S^{+}=1$, то операторы

$$
\widetilde{C}_{i}=S^{+} C_{i}^{0} S
$$

также являются решениями уравнения (29). Если $S$ - унитарный оператор, $S^{+} S=1$, то полевая конфигурация $\widetilde{C}_{i}$ является просто образом конфигурации $C_{i}^{0}$ при калибровочном преобразовании. Однако если $S$ - оператор частичной изометрии (см. (1)), то 
$\widetilde{C}_{i}$ дает новое решение уравнений поля. Например, начиная с вакуумной конфигурации, $u_{i}=0$, можно получить $n$-солитонное решение, используя операторы сдвига базиса

$$
S_{n}=\sum_{m}|m\rangle\langle m+n| .
$$

В частности, односолитонное решение имеет вид

$$
\widetilde{C}_{1}=\frac{i}{\theta} S_{1}^{+} \hat{p} S_{1}, \quad \widetilde{C}_{2}=-\frac{i}{\theta} S_{1}^{+} \hat{x} S_{1},
$$

или в терминах калибровочных полей

$$
\begin{aligned}
& u_{1}=\frac{1}{i}\left(S_{1}^{+} \partial_{1} S_{1}-\frac{i}{\theta} P_{0} \hat{p}\right), \\
& u_{2}=\frac{1}{i}\left(S_{1}^{+} \partial_{2} S_{1}+\frac{i}{\theta} P_{0} \hat{x}\right),
\end{aligned}
$$

где $P_{0}=|0\rangle\langle 0|$.

Легко убедиться в том, что прямо обобшить это построение на случай некоммутативного цилиндра не удается. Действительно, операторы $C_{i}$ не принадлежат алгебре некоммутативного цилиндра. Тем не менее можно попытаться воспользоваться вложением алгебры $\mathcal{A}_{\mathrm{C}}$ в алгебру $\mathcal{A}_{\mathrm{P}}$ и попробовать написать ту же формулу $(30)$, в которой оператор частичной изометрии $S^{\mathrm{C}}$ принадлежит теперь $\mathcal{A}_{\mathrm{C}}$ (см. (15)). Легко видеть, однако, что получаюшиеся калибровочные поля не принадлежат подалгебре $\mathcal{A}_{\mathrm{C}}$, поэтому такое построение не дает желаемого результата.

Для того чтобы получить метод генерации решений на некоммутативном цилиндре, обобшим формализм [1], связывающий метод генерации решений на некоммутативной плоскости с наличием изоморфизма между модулями $\mathcal{A}_{\mathrm{P}}$ и $\mathcal{A}_{\mathrm{P}} \oplus \mathcal{F}_{\mathrm{P}}$. Воспользуемся базисом, введенным в п. 2.1. Определим изоморфизм $\Lambda: \mathcal{A}_{\mathrm{C}} \rightarrow \mathcal{A}_{\mathrm{C}} \oplus \mathcal{F}_{\mathrm{C}}$ следуюшим образом:

$$
\Lambda: \hat{A} \rightarrow\left(\begin{array}{c}
\langle 0,0| \hat{A} \\
S_{1}^{\mathrm{C}} \hat{A}
\end{array}\right),
$$

где оператор $S_{1}^{\mathrm{C}}$ определен равенством (15). Обратное отображение задается формулой

$$
\Lambda^{-1}:\left(\begin{array}{c}
\langle f| \\
\hat{A}
\end{array}\right) \rightarrow S_{1}^{\mathrm{C}+} \hat{A}+\phi(\langle f|),
$$

где

$$
\phi(\langle f|)=\sum_{m}|0, m\rangle\langle f| e^{-2 \pi i R m \hat{p} / \theta} .
$$

Отметим следуюшее полезное свойство операции $\phi:$

$$
\phi(\langle f| \hat{A})=\phi(\langle f|) \hat{A}
$$


для любого $\hat{A} \in \mathcal{A}_{\mathrm{C}}$.

Заметим теперь, что связность (26) на прямой сумме $\mathcal{A}_{\mathrm{C}} \oplus \mathcal{F}_{\mathrm{C}}$ удовлетворяет уравнению (28). Используя изоморфизм $\Lambda$, можно определить связность $\nabla_{i}$ на свободном модуле $\mathcal{A}_{\mathrm{C}}$ :

$$
\nabla_{i}=\Lambda^{-1} \circ \widetilde{\nabla}_{i}^{0} \circ \Lambda .
$$

В случае некоммутативной плоскости аналогичное построение эквивалентно методу генерации решений [1]. В настоящем случае связность (35) также подчиняется уравнению поля (28), т.е. она соответствует солитону. Точное многосолитонное решение на некоммутативном цилиндре может быть получено многократным применением приведенной процедуры.

Вычислим теперь явно калибровочное поле односолитонного решения на некоммутативном цилиндре, получаюшееся в результате указанного построения. А именно, рассмотрим действие связности $\nabla_{i}$ на произвольный элемент $\hat{A} \in \mathcal{A}_{\mathrm{C}}$. Используя свойство (34), находим

$$
\nabla_{i}(\hat{A})=\left(\partial_{i}+S_{1}^{\mathrm{C}+} \partial_{i} S_{1}^{\mathrm{C}}-\phi\left(\langle 0,0| X_{i}\right)\right) \hat{A},
$$

где

$$
X_{1}=\frac{i}{\theta} \hat{p}, \quad X_{2}=-\frac{i}{\theta} \hat{x} .
$$

Поскольку $X_{1} \in \mathcal{A}_{\mathrm{C}}$, выражение для $\nabla_{1}$ можно упростить, еше раз воспользовавшись свойством (34). В результате для компоненты $u_{1}$ калибровочного поля получаем

$$
u_{1}=\frac{1}{i}\left(S_{1}^{\mathrm{C}+} \partial_{1} S_{1}^{\mathrm{C}}-\frac{i}{\theta} P_{0} \hat{p}\right) .
$$

Это выражение совпадает с выражением (31), верным в случае некоммутативной плоскости, если в качестве оператора частичной изометрии в равенство $(31)$ подставить $S_{1}^{\mathrm{C}}$.

Поскольку $\hat{x}$ не является элементом алгебры $\mathcal{A}_{\mathrm{C}}$, для $u_{2}$ нельзя написать выражение вида (32). Тем не менее формула (36) упрощается для базисов, принадлежащих определенному классу. А именно, слагаемое, содержашее $\phi$, в выражении для $u_{2}$ может быть переписано следуюшим образом:

$$
\begin{aligned}
\phi\left(\langle 0,0| X_{2}\right) & =\phi\left(\langle 0,0| P_{0} X_{2}\right)=\phi\left(\langle 0,0|\left[P_{0}, X_{2}\right]\right)+\phi\left(\langle 0,0| X_{2}\right) P_{0}= \\
& =P_{0}\left[P_{0}, X_{2}\right]+\sum_{m, l}|0, m\rangle\left\langle 0,0\left|X_{2}\right| 0, l\right\rangle\langle 0, l+m|,
\end{aligned}
$$

где в последнем равенстве мы использовали тот факт, что $\left[P_{0}, X_{2}\right] \in \mathcal{A}_{\mathrm{C}}$. Теперь легко проверить, что если базис имеет вид (19), а функции $\chi_{n}(\lambda)$ обладают определенной четностью (это свойство выполнено при выборе (20), (21)), то

$$
\left\langle n, 0\left|X_{2}\right| n, l\right\rangle=0
$$


для всех $n, l$ и последний член в выражении (37) обрашается в нуль. В этом случае находим следующее выражение для компоненты $u_{2}$ калибровочного поля:

$$
u_{2}=\frac{1}{i}\left(S_{1}^{\mathrm{C}+} \partial_{2} S_{1}^{\mathrm{C}}+P_{0} \partial_{2} P_{0}\right)
$$

Кривизна может быть выражена через проектор $P_{0}$ без использования какого-либо определенного выбора базиса и равна

$$
F_{12}=\left[\nabla_{1}, \nabla_{2}\right]=-\frac{i}{\theta} P_{0}
$$

как ясно из формул (35) и (26).

Энергия солитона равна

$$
E=-\frac{\pi \theta}{g^{2}} \operatorname{Tr}_{\mathrm{C}} F^{12} F_{12}=\frac{\pi}{g^{2} \theta} \operatorname{Tr}_{\mathrm{C}} P_{0}=\frac{\pi}{g^{2} \theta}
$$

что совпадает с энергией солитона на некоммутативной плоскости.

Кривизна $F_{12}$ и ее вейлевский символ не являются калибровочно-инвариантными величинами. Тем не менее форма солитона (в фиксированной калибровке) представляет определенный интерес. Вейлевский символ оператора $F_{12}$ на плоскости $(x, p)$ для $R=10$ и $\theta=3$, полученньй при помощи базиса (20), (21), показан на рисунке (вычисления, приводящие к этому графику, описаны в приложении). Периодичность по координате $x$ отражает тот факт, что символ соответствует солитону на цилиндре.

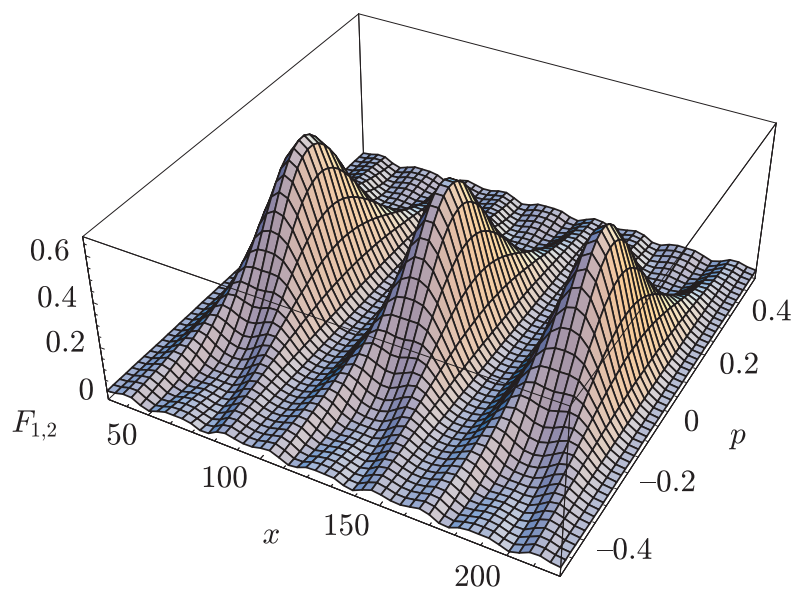




\section{4. СПЕКТР ФЛУКТУАЦИЙ}

Для исследования спектра возмушений вокруг солитона, найденного в разделе 3 , удобно, следуя [10], использовать изоморфизм $\Lambda$, определенный соотношением (33), и выразить функционал действия для возмущений на фоне солитона в терминах связностей на прямой сумме $\mathcal{A}_{\mathrm{C}} \oplus \mathcal{F}_{\mathrm{C}}$. Первый шаг состоит в определении понятия следа эндоморфизма этого модуля. Для произвольного эндоморфизма, имеюшего вид второго слагаемого в (25), определим

$$
\operatorname{Tr}_{\mathcal{A} \oplus \mathcal{F}}\left(\begin{array}{cc}
z_{i} & \psi_{i} \\
\psi_{i}^{*} & v_{i}
\end{array}\right)=\operatorname{Tr}_{\mathrm{C}}\left(P_{0} z_{i}\right)+\operatorname{Tr}_{\mathrm{C}} v_{i}
$$

Можно непосредственно проверить, что определенный таким образом след совпадает со следом, индуцируемым из $\operatorname{Tr}_{\mathrm{C}}$ при изоморфизме $\Lambda$.

Мы используем следуюшее обозначение:

$$
\operatorname{Tr} \psi_{2} \psi_{1}^{*}=\left\langle\psi_{1} \mid \psi_{2}\right\rangle
$$

где векторы $\left|\psi_{i}\right\rangle$ определены в (27). Действие для возмущений на фоне солитона в калибровке $A_{0}=0$ принимает вид

$$
S=\frac{\pi \theta}{2 g^{2}} \operatorname{Tr}_{\mathcal{A} \oplus \mathcal{F}} \int d t\left(-2\left[\partial_{t}, \nabla_{i}\right]^{2}+\left[\nabla_{i}, \nabla_{j}\right]^{2}\right),
$$

где $\nabla_{i}=\widetilde{\nabla}_{i}^{0}+i \delta A_{i}$. Здесь $\delta A_{i}-$ эрмитова матрица, описываюшая флуктуации:

$$
\delta A_{i}=\left(\begin{array}{cc}
z_{i} & \psi_{i} \\
\psi_{i}^{*} & v_{i}
\end{array}\right)
$$

Квадратичная часть действия может быть записана в виде суммы вкладов от трех невзаимодействующих секторов. Первый сектор содержит поля $v_{i}$ (в терминологии теории струн этот сектор описывает $(2,2)$-струны - открытые струны, у которых оба конца двигаются свободно вдоль $D 2$-браны, заполняющей цилиндр), второй сектор содержит поля $z_{i}$ (соответствующие $(0,0)$-струнам, оба конца которых закреплены на $D 0$-бране (солитоне)), и, наконец, третий сектор содержит внедиагональные компоненты $\psi, \psi^{*}$ $((0,2)$-струны, один конец которых движется вдоль $D 2$-браны, а другой закреплен на $D 0$-бране).

Квадратичное действие для первого сектора имеет следуюший вид:

$$
S_{v}=\frac{\pi \theta}{2 g^{2}} \operatorname{Tr}_{\mathrm{C}} \int d t\left(2\left(\partial_{0} v_{i}\right)^{2}-2\left(\partial_{1} v_{2}-\partial_{2} v_{1}\right)^{2}\right) .
$$

Это выражение совпадает с квадратичной частью действия $U(1)$-калибровочной теории на некоммутативном цилиндре без солитона, записанной в калибровке $u_{0}=0$. На квадратичном уровне коммутативная и некоммутативная теории совпадают, поэтому 
спектр в $(2,2)$-секторе является просто спектром абелевой калибровочной теории на цилиндре.

В $(0,0)$-секторе на квадратичном уровне имеем

$$
S_{z}=\frac{2 \pi \theta}{g^{2}} \int d t \sum_{n=0}^{\infty}\left[\left|\partial_{t} z_{i}(n)\right|^{2}-4 \pi^{2} \frac{R^{2}}{\theta^{2}} n^{2}\left|z_{1}(n)\right|^{2}\right]
$$

где $z_{i}(n)$ определены равенством (24) и мы воспользовались условием эрмитовости

$$
z_{i}(-n)=z_{i}(n)^{*}
$$

Этим действием описываются набор массивных мод $z_{1}(n), n=1,2, \ldots$, локализованных на солитоне и имеющих массы

$$
m_{n}=2 \pi n \frac{R}{\theta}
$$

и два безмассовых модуля $z_{i}(0)$ с $n=0$, соответствуюшие положению солитона на некоммутативном цилиндре. Все моды $z_{2}(n) \mathrm{c} n \neq 0$ являются нефизическими и устраняются наложением условия Гаусса, имеюшего в $(0,0)$-секторе следуюший вид:

$$
\partial_{0} \partial_{i} z_{i}=0
$$

Стоит отметить, что массы (39) пропорциональны радиусу цилиндра; следовательно, физическим массивным модам $z_{1}(n)$ в теории струн соответствуют струны с нетривиальной намоткой на цилиндр.

Наконец, во внедиагональном секторе приходим к следуюшему действию:

$$
S_{\psi}=\frac{2 \pi \theta}{g^{2}} \int d t\left(\operatorname{Tr} \partial_{0} \psi_{i}^{*} \partial_{0} \psi_{i}-\frac{1}{\theta}\left(\| a\left|\psi_{-}\right\rangle-a^{+}\left|\psi_{+}\right\rangle \|^{2}-\left\langle\psi_{-} \mid \psi_{-}\right\rangle+\left\langle\psi_{+} \mid \psi_{+}\right\rangle\right)\right)
$$

где мы ввели новые поля

$$
\psi_{1}=\frac{i}{\sqrt{2}}\left(\psi_{-}-\psi_{+}\right), \quad \psi_{2}=\frac{1}{\sqrt{2}}\left(\psi_{-}+\psi_{+}\right)
$$

и операторы рождения и уничтожения

$$
a^{+}=\frac{1}{\sqrt{2 \theta}}(\hat{x}+i \hat{p}), \quad a=\frac{1}{\sqrt{2 \theta}}(\hat{x}-i \hat{p}) .
$$

Раскладывая

$$
\left|\psi_{ \pm}\right\rangle=\sum_{n=0}^{\infty} t_{n}^{ \pm}|n\rangle,
$$


где $|n\rangle$ - собственные состояния осциллятора, и подставляя это выражение в действие, получаем следующее выражение для потенциального члена в действии (40):

$$
-\frac{4}{\theta}\left(\sum_{n=1}^{\infty}\left|t_{n+1}^{-} \sqrt{n}-t_{n-1}^{+} \sqrt{n+1}\right|^{2}-\left|t_{0}^{-}\right|^{2}\right) .
$$

Эта массовая матрица диагонализуется преобразованием

$$
\begin{aligned}
& \varphi_{n}=\frac{1}{\sqrt{2 n+1}}\left(t_{n+1}^{-} \sqrt{n}-t_{n-1}^{+} \sqrt{n+1}\right), \\
& \eta_{n}=\frac{1}{\sqrt{2 n+1}}\left(t_{n+1}^{-} \sqrt{n+1}+t_{n-1}^{+} \sqrt{n}\right) .
\end{aligned}
$$

Окончательно получаем

$$
S_{\psi}=\frac{2 \pi \theta}{g^{2}} \int d t\left[\left|\partial_{0} t_{0}^{-}\right|^{2}+\frac{1}{\theta}\left|t_{0}^{-}\right|^{2}+\left|\partial_{0} \eta_{0}\right|^{2}+\sum_{n=1}^{\infty}\left(\left|\partial_{0} \varphi_{n}\right|^{2}+\left|\partial_{0} \eta_{n}\right|^{2}-\frac{2 n+1}{\theta}\left|\varphi_{n}\right|^{2}\right)\right] .
$$

Условие Гаусса в этом секторе имеет вид

$$
\partial_{0}\left(\nabla_{i}^{0} \psi_{i}\right)=0
$$

откуда следует, что все поля $\eta_{n}$ являются нефизическими. Среди физических полей есть одна тахионная мода $t_{0}^{-}$с квадратом массы $-1 / \theta$ и набор массивных полей $\phi_{n}, \quad n=$ $1,2, \ldots$, со спектром масс $m_{n}^{2}=(2 n+1) / \theta$.

Все спектры, вычисленные в данном разделе, согласуются со спектрами различных секторов теории струн на цилиндре. Этим подтверждается интерпретация некоммутативных солитонов в качестве $D$-бран.

\section{5. ОБСУЖДЕНИЕ}

Подведем итоги. Ключевым моментом в данной статье является явное построение ортонормированного базиса $|n, m\rangle$ в гильбертовом пространстве функций от одной переменной. Элементы этого базиса преобразуются по правилу (10) при действии на них оператора трансляции. Это свойство позволяет построить полный набор ортогональных проекторов (12) и соответствующий набор операторов частичной изометрии (15) в алгебре $\mathcal{A}_{\mathrm{C}}$ некоммутативного цилиндра. Используя эти операторы частичной изометрии, мы установили изоморфизм (33) между свободным модулем над алгеброй $\mathcal{A}_{\mathrm{C}}$ и прямой суммой $\mathcal{F}_{\mathrm{C}} \oplus \mathcal{A}_{\mathrm{C}}$ фоковского модуля $\mathcal{F}_{\mathrm{C}}$ и свободного модуля. Это построение привело к обобшению метода генерации решений на случай некоммутативного цилиндра. Мы описали явно калибровочное поле односолитонного решения и вычислили спектр малых возмушений над этим решением. 
В заключение обсудим другое следствие существования базиса $|n, m\rangle$. В работе [15] было отмечено следующее необычное свойство калибровочной теории на некоммутативной плоскости. Рассмотрим $U(N)$-калибровочную теорию с некоторым $N$ на некоммутативной плоскости. Тогда для любого натурального $K$ в этой теории существует вакуум такой, что теория над этим вакуумом совпадает с $U(K)$-калибровочной теорией над тривиальным вакуумом. Интерпретация этого свойства, данная в [15], состоит в том, что число цветов $N$ является параметром суперотбора, нумерующим различные секторы в квантовом гильбертовом пространстве некоммутативной калибровочной теории.

С технической точки зрения описанное выше свойство обусловлено в случае некоммутативной плоскости сушествованием изоморфизма между суммами свободных модулей $\sum_{1}^{N} \mathcal{A}_{\mathrm{P}}$ и $\sum_{1}^{K} \mathcal{A}_{\mathrm{P}}$ с любыми $N$ и $K$. Калибровочные теории на этих суммах являются $U(N)$ - и $U(K)$-теориями, соответственно, так что этот изоморфизм порождает связность, соответствуюшую $U(K)$-вакууму в $U(N)$-калибровочной теории.

В случае некоммутативного тора это свойство, по-видимому, отсутствует, так как прямые суммы $\sum_{1}^{N} \mathcal{A}_{\mathrm{T}}$ и $\sum_{1}^{K} \mathcal{A}_{\mathrm{T}}$ с разными $N$ и $K$ не изоморфны [1]. Покажем, что из свойств базиса $|n, m\rangle$ следует, что некоммутативный цилиндр аналогичен в этом смысле некоммутативной плоскости: $U(N)$-калибровочные теории с разными $N$ возникают на некоммутативном цилиндре как теории над разными вакуумами в единой калибровочной теории.

Для простоты рассмотрим, как $U(2)$-калибровочная теория возникает в $U(1)$-калибровочной теории (обобщение на случай других $N$ и $K$ может быть проведено непосредственно). Сначала построим изоморфизм между модулями $\mathcal{A}_{\mathrm{C}}$ и $\mathcal{A}_{\mathrm{C}} \oplus \mathcal{A}_{\mathrm{C}}$. Элементами прямой суммы $\mathcal{A}_{\mathrm{C}} \oplus \mathcal{A}_{\mathrm{C}}$ являются столбцы

$$
\left(\begin{array}{c}
\hat{A}_{1} \\
\hat{A}_{2}
\end{array}\right)
$$

где $\hat{A}_{1}$ и $\hat{A}_{2}$ - элементы алгебры $\mathcal{A}_{\mathrm{C}}$. Определим отображение $\Lambda: \mathcal{A}_{\mathrm{C}} \rightarrow \mathcal{A}_{\mathrm{C}} \oplus \mathcal{A}_{\mathrm{C}}$ следуюшим образом:

$$
\Lambda: \hat{A} \rightarrow\left(\begin{array}{c}
E \hat{A} \\
O \hat{A}
\end{array}\right)
$$

где операторы $E$ и $O$ имеют вид

$$
E=\sum_{m, n}|n, m\rangle\left\langle m, 2 n\left|, \quad O=\sum_{m, n}\right| n, m\right\rangle\langle m, 2 n+1| .
$$

Непосредственно проверяется, что эти операторы обладают следуюшими свойствами:

$$
O O^{+}=E E^{+}=O^{+} O+E^{+} E=1, \quad E O^{+}=O E^{+}=0 .
$$

Используя эти свойства, убеждаемся, что обратное отображение $\Lambda^{-1}: \mathcal{A}_{\mathrm{C}} \oplus \mathcal{A}_{\mathrm{C}} \rightarrow \mathcal{A}_{\mathrm{C}}$ имеет вид

$$
\Lambda^{-1}:\left(\begin{array}{c}
\hat{A}_{1} \\
\hat{A}_{2}
\end{array}\right) \rightarrow E^{+} \hat{A}_{1}+O^{+} \hat{A}_{2}
$$


Таким образом, $\Lambda$ является изоморфизмом. Так же как в разделе 3 , построим связность на модуле $\mathcal{A}_{\mathrm{C}}$

$$
\nabla_{i}=\Lambda^{-1} \circ \partial_{i} \circ \Lambda
$$

индуцированную из вакуумной связности $\partial_{i}$ на модуле $\mathcal{A}_{\mathrm{C}} \oplus \mathcal{A}_{\mathrm{C}} . U(1)$-калибровочное поле, соответствуюшее связности $\nabla_{i}$, вычисляется непосредственно:

$$
A_{i}=E^{+} \partial_{i} E+O^{+} \partial_{i} O .
$$

По построению кривизна этого калибровочного поля равна нулю, и $U(1)$-теория на некоммутативном цилиндре на фоне (41) совпадает с $U(2)$-теорией на некоммутативном цилиндре на тривиальном фоне.

Таким образом, любая $U(N)$-калибровочная теория как на некоммутативной плоскости, так и на некоммутативном цилиндре обладает бесконечным набором вакуумов с номерами $K=1,2, \ldots$. Над каждым из этих вакуумов теория эквивалентна $U(K)$-калибровочной теории над ее тривиальным вакуумом. Естественно поставить вопрос о том, соответствуют ли эти вакуумы различным секторам суперотбора или же они просто являются разными фазами одной и той же теории, как, например, вырожденные вакуумы с различными вакуумными средними значениями скалярного поля в (коммутативных) скалярных теориях. В принципе теория на некоммутативном цилиндре наиболее адекватна для анализа этого вопроса: в последнем случае должны существовать конфигурации типа доменных стенок (не обязательно решения) с конечной әнергией, разделяюшие разные вакуумы. Было бы интересно построить такие конфигурации либо доказать их отсутствие.

\section{Вейлевский символ $F_{12}$}

ПРИЛОЖЕНИЕ

Вычислим вейлевский символ кривизны (38), полученный при помоши базиса, заданного формулами (20), (21). Для простоты положим $R=\theta=1$. Из выражения (12) следует, что ядро проектора $P_{0}$ имеет вид

$$
P_{0}\left(p, p^{\prime}\right)=\sum_{m} \xi_{1}(p) \xi_{1}\left(p^{\prime}\right) e^{2 \pi i m\left(p-p^{\prime}\right)} .
$$

Таким образом, для коэффициентных функций $a(p, q)($ cp. с (5)) находим

$$
a(p, q)=\xi_{1}(p) \xi_{1}(p-q) .
$$

Используя формулу (8), запишем

$$
\tilde{f}_{P_{0}}(m, p)=2 \pi a\left(p-\frac{m}{2},-m\right)
$$


где фурье-образ $\tilde{f}_{P_{0}}(m, p)$ вейлевского символа определен в $(9)$. Тогда выражение для самого вейлевского символа принимает вид

$$
\begin{aligned}
f(x, p)= & \sum_{n} \xi_{1}\left(p-\frac{n}{2}\right) \xi_{1}\left(p+\frac{n}{2}\right) e^{-i n x}= \\
= & \frac{\cos 2 \pi p}{2 \pi^{2} p} \sum_{n=-\infty}^{n=\infty} \cos n x\left(\frac{1}{n^{2}-(2 p+1)^{2}}-\frac{1}{n^{2}-(2 p-1)^{2}}\right)+ \\
& +\frac{1}{2 \pi^{2} p} \sum_{n=-\infty}^{n=\infty} \cos n x\left(\frac{(-1)^{n}}{n^{2}-(2 p+1)^{2}}-\frac{(-1)^{n}}{n^{2}-(2 p-1)^{2}}\right) .
\end{aligned}
$$

В результате вычисления сумм получаем следующие явные выражения для вейлевского символа $f_{P_{0}}(x, p)$ в различных интервалах значений аргумента (мы восстановили зависимость от $R$ и $\theta$ в окончательных формулах):

1) при $x \in[0, \pi R]$

$$
\begin{aligned}
f_{P_{0}}(x, p)= & \frac{1}{\theta}\left[\frac { \operatorname { c o s } ( 2 \pi R p / \theta ) } { 2 \pi ^ { 2 } R p / \theta } \left(\frac{\pi}{2 R p / \theta-1} \frac{\cos [(\pi-x / R)(2 R p / \theta-1)]}{\sin (\pi(2 R p / \theta-1))}-\right.\right. \\
& \left.-\frac{\pi}{2 R p / \theta+1} \frac{\cos [(\pi-x / R)(2 R p / \theta+1)]}{\sin (\pi(2 R p / \theta+1))}\right)+ \\
& +\frac{1}{2 \pi^{2} R p / \theta}\left(\frac{\pi}{2 R p / \theta-1} \frac{\cos (2 x p / \theta-x / R)}{\sin (\pi(2 R p / \theta-1))}-\right. \\
& \left.\left.-\frac{\pi}{2 R p / \theta+1} \frac{\cos (2 p x / \theta+x / R)}{\sin (\pi(2 R p / \theta+1))}\right)\right]
\end{aligned}
$$

2) при $x \in[\pi R, 2 \pi R]$

$$
\begin{aligned}
f_{P_{0}}(x, p)= & \frac{1}{\theta}\left[\frac { \operatorname { c o s } ( 2 \pi R p / \theta ) } { 2 \pi ^ { 2 } R p / \theta } \left(\frac{\pi}{2 R p / \theta-1} \frac{\cos [(\pi-x / R)(2 R p / \theta-1)]}{\sin (\pi(2 R p / \theta-1))}-\right.\right. \\
& \left.-\frac{\pi}{2 R p / \theta+1} \frac{\cos [(\pi-x / R)(2 R p / \theta+1)]}{\sin (\pi(2 R p / \theta+1))}\right)+ \\
& +\frac{1}{2 \pi^{2} R p / \theta}\left(\frac{\pi}{2 R p / \theta-1} \frac{\cos [(2 \pi-x / R)(2 R p / \theta-1)]}{\sin (\pi(2 R p / \theta-1))}-\right. \\
& \left.\left.-\frac{\pi}{2 R p / \theta+1} \frac{\cos [(2 \pi-x / R)(2 R p / \theta+1)]}{\sin (\pi(2 R p / \theta+1))}\right)\right]
\end{aligned}
$$

Эта функция неаналитична в точке $x=\pi R$, однако можно явно проверить, что $f_{P_{0}}$ и ее производная в этой точке непрерывны.

Благодарности. Авторы глубоко благодарны А. Конечному за полезные обсуждения. Работа выполнена при поддержке Российского фонда фундаментальных исследований (гранты № 02-02-17398, 02-02-06514, 02-02-06515) и Swiss Science Foundation (грант № 7SUPJ062239). Работа С. Дубовского частично поддержана INTAS (грант № YS 2001-2/128). Работа С. Сибирякова частично поддержана INTAS (грант № YS 2001-2/141). 


\section{Список литературы}

[1] A. Konechny, A. Schwarz. Phys. Rep. 2002. V. 360. P. 353; hep-th/0012145; P. 465; hep-th/0107251.

[2] J. A. Harvey. Komaba lectures on noncommutative solitons and D-branes. hep-th/0102076.

[3] M.R. Douglas, N. A. Nekrasov. Rev. Mod. Phys. 2001. V. 73. P. 977; hep-th/0106048.

[4] I. Y. Aref'eva, D. M. Belov, A. A. Giryavets, A.S. Koshelev, P. B. Medvedev. Noncommutative field theories and (super)string field theories. hep-th/0111208.

[5] N. Seiberg, E. Witten. JHEP. 1999. V. 9909. P. 032; hep-th/9908142.

[6] J.A. Harvey, P. Kraus, F. Larsen, E.J. Martinec. JHEP. 2000. V. 0007. P. 042; hep-th/0005031.

[7] A.P. Polychronakos. Phys. Lett. B. 2000. V. 495. P. 407; hep-th/0007043; D. Bak. Phys. Lett. B. 2000. V. 495. P. 251; hep-th/0008204; M. Aganagic, R. Gopakumar, S. Minwalla, A. Strominger. JHEP. 2001. V. 0104. P. 001; hep-th/0009142.

[8] R. Gopakumar, S. Minwalla, A. Strominger. JHEP. 2000. V. 0005. P. 020; hep-th/0003160.

[9] J. A. Harvey, P. Kraus, F. Larsen. JHEP. 2000. V. 0012. P. 024; hep-th/0010060.

[10] A. Konechny. JHEP. 2002. V. 0203. P. 035; hep-th/0112189.

[11] R. Gopakumar, M. Headrick, M. Spradlin. On noncommutative multi-solitons. hep-th/ 0103256 .

[12] M. Chaichian, A. Demichev, P. Presnajder, A. Tureanu. Eur. Phys. J. C. 2001. V. 20. P. 767; hep-th/0007156; M. Chaichian, A. Demichev, P. Presnajder, M. M. Sheikh-Jabbari, A. Tureanu. Nucl. Phys. B. 2001. V. 611. P. 383; hep-th/0101209.

[13] D. Bak, K. M. Lee. Phys. Lett. B. 2001. V. 509. P. 168; hep-th/0103148.

[14] M. Hamanaka. Phys. Rev. D. 2002. V. 65. P. 085022; hep-th/0109070.

[15] D. J. Gross, N. A. Nekrasov. JHEP. 2001. V. 0103. P. 044; hep-th/0010090. 